\title{
Neumonía grave por Streptococcus pyogenes. Reporte de un caso
}

\author{
Fernando Saldías $P$, Jorge Yáñez V, Velia Saldías $H^{a}$, \\ O rlando Díaz $\mathbf{P}$.
}

\section{Community-acquired pneumonia caused by Streptococcus pyogenes. Report of one case}

During the past two decades there has been a resurgence of invasive group A streptococcal (GAS) infection, specially pneumonia and bacteremia. We report a 35 yearold female previously subjected to a thyroidectomy for a thyroid cancer, that five days after operation, presented with a severe community-acquired pneumonia caused by Streptococcus pyogenes (Lancefield Group A Streptococcus) that was complicated by acute respiratory failure and septic shock. She was treated with a combination of $3 \mathrm{~g} /$ day of cefotaxime and $1.8 \mathrm{~g} /$ day of clindamycin with a good clinical response and discharged from the hospital in good conditions. Although this microorganism is an uncommon cause of community-acquired pneumonia, previously healthy individuals may be infected and the clinical course may be fulminant. Patients with invasive GAS infection admitted to ICU have a high mortality rate. Treatment of choice of Group A streptococcal infection is penicillin. However, clindamycin should be added in severe infections (Rev Méd Chile 2008; 136: 1564-9).

(Key words: Clindamycin; Pneumonia, bacterial; Streptococcus pyogenes)

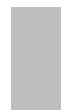

Recibido el 10 de diciembre, 2007. Aceptado el 20 de junio, 2008.

Departamento de Enfermedades Respiratorias, Pontificia Universidad Católica de Chile.

aAlumna $7^{\circ}$ Año de Medicina, Universidad de Los Andes, Santiago de Chile.

$\mathrm{E}^{1}$ Streptococcus pyogenes o estreptococo ßhemolítico del grupo A de Lancefield es uno de los patógenos bacterianos más importante que afecta a los seres humanos ${ }^{1}$. Es el principal agente bacteriano aislado en faringitis aguda, ocasionalmente es el agente causal de neumonía o bacteriemia, generalmente asociado a infecciones virales

$\overline{\text { Correspondencia a: Dr. Fernando Saldías Peñafiel. Departa- }}$ mento de Enfermedades Respiratorias, Pontificia Universidad Católica de Chile. Marcoleta 352 - $1^{\text {er }}$ Piso, Santiago, Chile. Teléfonos: 6331541 - 3543242. Fax: 6335255.

E mail: fsaldias@med.puc.cl (influenza, sarampión) o coqueluche ${ }^{2}$. La neumonía por Streptococcus pyogenes es poco frecuente en el adulto y se ve algo más en el niño, en quienes puede ocasionar compromiso parenquimatoso pulmonar y en oportunidades derrame pleural purulento ${ }^{3}$.

A comienzos del siglo XX, se estimaba que ocasionaba $2 \%-5 \%$ de los casos de neumonía lobar, las complicaciones supurativas como el empiema eran frecuentes y la letalidad era elevada (cercana a $50 \%)^{4}$. Se reportaron varios brotes en los regimientos, especialmente cuando la infección estreptocócica faríngea y escarlatina eran prevalentes en la comunidad ${ }^{5,6}$. Se trataba de 
población joven saludable, la tasa de bacteriemia era baja y la letalidad disminuyó significativamente con el advenimiento de los antibióticos. En ocasiones, el cuadro clínico puede ser muy severo, complicando a la neumonía un shock séptico causado por el microorganismo, cuyo curso clínico puede ser fulminante ${ }^{7}$. La asociación de shock y bacteriemia por S pyogenes es inusual, reportándose una prevalencia de 0,18 episodios por 1.000 admisiones hospitalarias ${ }^{8}$.

En la actualidad, se han descrito algunos brotes en centros geriátricos y casos esporádicos en la comunidad, afectando a pacientes con enfermedades crónicas tales como diabetes, neoplasias, alcoholismo e inmunosupresión ${ }^{9,10}$. El riesgo de muerte aumenta en los adultos mayores y en las infecciones bacteriémicas ${ }^{4}$. En los últimos 15 años han resurgido formas graves de la enfermedad, se ha comunicado un aumento de la prevalencia de neumonía y fasceítis necrotizante, ocasionalmente asociado a síndrome de shock tóxico ${ }^{9-12}$. Presentamos el caso clínico de una paciente con neumonía bacteriémica complicada con shock séptico, en quien se aisló Streptococcus pyogenes.

\section{Caso CLÍNICO}

Paciente de 35 años, sexo femenino, no fumadora, fue sometida a tiroidectomía total el 20 de agosto de 2007 por neoplasia folicular de tiroides y fue dada de alta en buenas condiciones generales a las 48 h. Consultó el 25 de agosto por malestar general, odinofagia, cefalea, sensación febril, escalofríos, dolor cervical, tos productiva y expectoración mucopurulenta, siendo admitida a sala de cuidados generales. En el examen físico destacaba paciente en regulares condiciones generales, lúcida, orientada, FC: 90 lat/min, PA: 106/60 mmHg, FR: $24 \mathrm{resp} / \mathrm{min}, \mathrm{T}: 37,8^{\circ} \mathrm{C}$. Dolor a la palpación en la zona de herida operatoria de la región cervical anterior, faringe sana, examen cardiopulmonar y abdominal normales. Se planteó el diagnóstico de infección de herida operatoria y se inició tratamiento con ceftriaxona $1 \mathrm{~g}$ /día EV. La paciente evolucionó febril y con dificultad respiratoria progresiva, requiriendo aporte de oxígeno al $35 \%$ para corregir el trastorno del intercambio gaseoso $\left(\mathrm{PaO}_{2} / \mathrm{FiO}_{2}:\right.$ 259). En el hemograma destacaba leucopenia $\left(2.700 / \mathrm{mm}^{3}\right)$ con desviación a izquierda (13\% baciliformes), PCR: $5,4 \mathrm{mg} / \mathrm{dL}$, GSA: pH: 7,46, $\mathrm{PaO}_{2}: 54,4 \mathrm{mmHg}, \mathrm{SaO}_{2}: 89 \%$, $\mathrm{PaCO}_{2}: 27,5 \mathrm{mmHg}, \quad$ B. act: $19,6 \mathrm{mEq} / \mathrm{L}$. La radiografía de tórax mostró opacidades parenquimatosas en lóbulo superior derecho, lóbulo medio, língula y lóbulo inferior izquierdo (Figura 1). La condición clínica de la paciente rápidamente se deterioró, con aumento de la dificultad respiratoria y deterioro del intercambio gaseoso (FR: 32 resp/min, $\mathrm{FiO}_{2}: 50 \%$ para lograr $\mathrm{SaO}_{2}>90 \%$ )

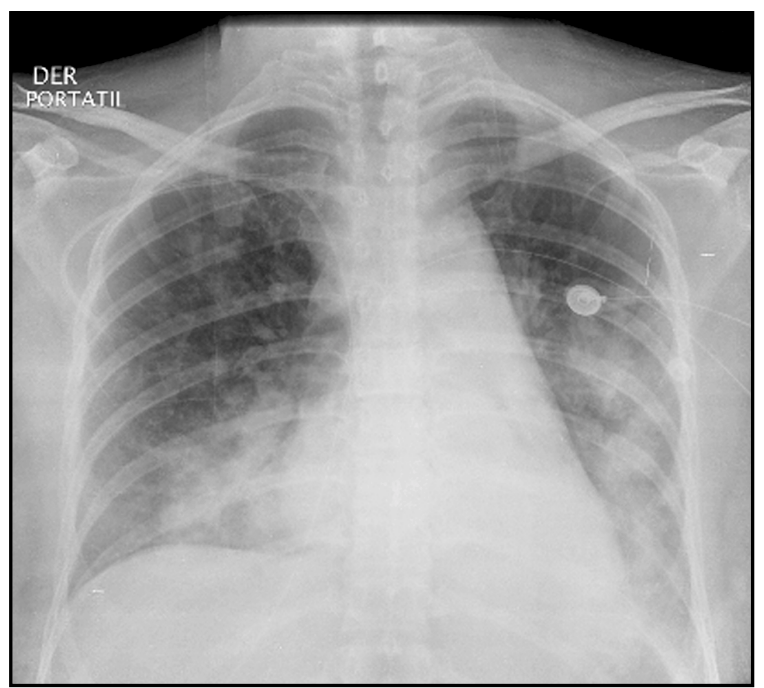

Figura 1. Radiografía de tórax muestra opacidades parenquimatosas bilaterales de predominio en ambos lóbulos inferiores. 
asociado a compromiso hemodinámico (hipotensión arterial que no respondió al aporte de volumen), trasladándose ese mismo día a la Unidad de Cuidados Intensivos, donde se constató paciente somnolienta, bradipsíquica, con llene capilar lento, FC: 130 lat/min, PA: 118/45, FR: 36 resp/min, $\mathrm{SaO}_{2}:$ 91\% con $\mathrm{FiO}_{2}$ : 1,0. APACHE de ingreso: 18. En la Tabla 1 se describe la evolución de los exámenes de laboratorio. La radiografía de tórax mostró progresión de los infiltrados pulmonares bilaterales en ocho horas. La paciente fue intubada y conectada a ventilador mecánico (VM). La tomografía computarizada cervical descartó colección supurada en zona operatoria y la tomografía axial compuratizada (TAC) de tórax mostró opacidades difusas en el parénquima pulmonar, especialmente en ambos lóbulos inferiores, y derrame pleural bilateral compatibles con el diagnóstico de neumonía grave y síndrome de distress respiratorio del adulto (SDRA) (Figura 2). La paciente fue manejada con aporte de volumen, noradrenalina en infusión continua $(0,15 \mathrm{\gamma} / \mathrm{kg} / \mathrm{min})$, antibióticos de amplio espectro (tazonam 4,5 g cada $8 \mathrm{~h}$, vancomicina $1 \mathrm{~g}$ cada $12 \mathrm{~h}$ y levofloxacina $500 \mathrm{mg}$ cada $12 \mathrm{~h}$ ). La paciente evolucionó con inestabilidad hemodinámica durante $72 \mathrm{~h}$, requiriendo apoyo con noradrenalina en infusión continua, $\mathrm{PaO}_{2} / \mathrm{FIO}_{2}$ alrededor de 200, y al tercer día se confirmó el aislamiento de Streptococcus pyogenes en los hemocultivos, sensible a penicilina, eritromicina y cefotaxima, por lo cual se modificó la terapia antibiótica y se cambió a cefotaxima $1 \mathrm{~g}$ cada 8 h y clindamicina $600 \mathrm{mg}$ cada $8 \mathrm{~h} \mathrm{EV}$. A las 72 h, se estabilizó la hemodinamia y se retiró el apoyo de drogas vasoactivas, y el 28 de agosto se desconectó del ventilador; sin embargo, la paciente evolucionó con gran apremio respiratorio, aumento de los requerimientos de oxígeno y signos de fatiga respiratoria, debiendo reconectarse al ventilador mecánico, siendo apoyada con presión de soporte y PEEP durante 3 días. La evolución clínica fue favorable, se mantuvo bien adaptada a VM con bajos requerimientos de oxígeno, hemodinamia estable y se extubó sin incidentes el 31 de agosto. La paciente no presentó nuevas complicaciones en la evolución, siendo

Tabla 1. Exámenes de laboratorio de paciente con neumonía comunitaria grave por Streptococcus pyogenes

\begin{tabular}{|c|c|c|c|c|c|c|c|}
\hline Fecha & $25-08-07$ & $25-08-07$ & $25-08-07$ & $26-08-07$ & $27-08-07$ & $28-08-07$ & $30-08-07$ \\
\hline Hora & $9: 00 \mathrm{~h}$ & $14: 00 \mathrm{~h}$ & $18: 30 \mathrm{~h}$ & $7: 00 \mathrm{~h}$ & $7: 00 \mathrm{~h}$ & $7: 00 \mathrm{~h}$ & $7: 00 \mathrm{~h}$ \\
\hline $\mathrm{FiO}_{2}$ & 0,21 & 1,0 & 0,70 & 0,30 & 0,40 & 0,30 & 0,30 \\
\hline $\mathrm{pH}^{2}$ & 7,46 & 7,42 & 7,36 & 7,36 & 7,39 & 7,42 & 7,51 \\
\hline $\mathrm{PaO}_{2}(\mathrm{mmHg})$ & 54,4 & 83 & 88 & 79,1 & 96,9 & 75 & 83,9 \\
\hline $\mathrm{SaO}_{2}(\%)$ & 89 & 96 & 96 & 95 & 97 & 95 & 96 \\
\hline $\mathrm{PaCO}_{2}(\mathrm{mmHg})$ & 27,5 & 30,3 & 31 & 35,2 & 31,1 & 28 & 37,8 \\
\hline $\mathrm{HCO}_{3}^{-}(\mathrm{mEq} / \mathrm{L})$ & 19,6 & 19,9 & 17,5 & 20,0 & 18,8 & 18,2 & 30,1 \\
\hline Lactato $(\mathrm{mmol} / \mathrm{L})$ & 5 & 3 & 2 & 2,1 & 2,2 & 1,4 & 0,9 \\
\hline Hematocrito (\%) & 39,6 & & & 33,4 & 27,4 & 28,7 & 28,0 \\
\hline Leucocitos $\left(\mathrm{mm}^{3}\right)$ & 2.700 & & & 9.800 & 17.000 & 20.700 & 17.400 \\
\hline Segmentados-baciliformes (\%) & $63-13$ & & & & $72-25$ & & \\
\hline PCR (mg/dL) & 5,4 & 8,5 & & 29,6 & 33,6 & 30,4 & 9,4 \\
\hline $\mathrm{Na}^{+}(\mathrm{mEq} / \mathrm{L})$ & 137 & & & 142 & 144 & 147 & 149 \\
\hline $\mathrm{K}^{+}(\mathrm{mEq} / \mathrm{L})$ & 3,0 & & & 3,8 & 3,2 & 3,3 & 3,5 \\
\hline $\mathrm{Cl}^{-}(\mathrm{mEq} / \mathrm{L})$ & 106 & & & 111 & 115 & 116 & 115 \\
\hline BUN (mg/dL) & 12 & & & 17 & 19 & 15 & 25 \\
\hline Creatininemia (mg/dL) & 0,63 & & & 0,68 & 0,61 & 0,53 & 0,44 \\
\hline Albúmina $(\mathrm{g} / \mathrm{dL})$ & 4,1 & & & & 2,0 & 2,4 & 2,5 \\
\hline Glicemia (mg/dL) & 124 & & & & 89 & & \\
\hline Protrombina (\%) & 43 & & & & 35 & 52 & 55 \\
\hline TTPA (seg) & 46,1 & & & & 41,8 & 33,2 & \\
\hline Plaquetas $\left(\mathrm{mm}^{3}\right)$ & 214.000 & & & & 167.000 & 166.000 & 124.000 \\
\hline
\end{tabular}




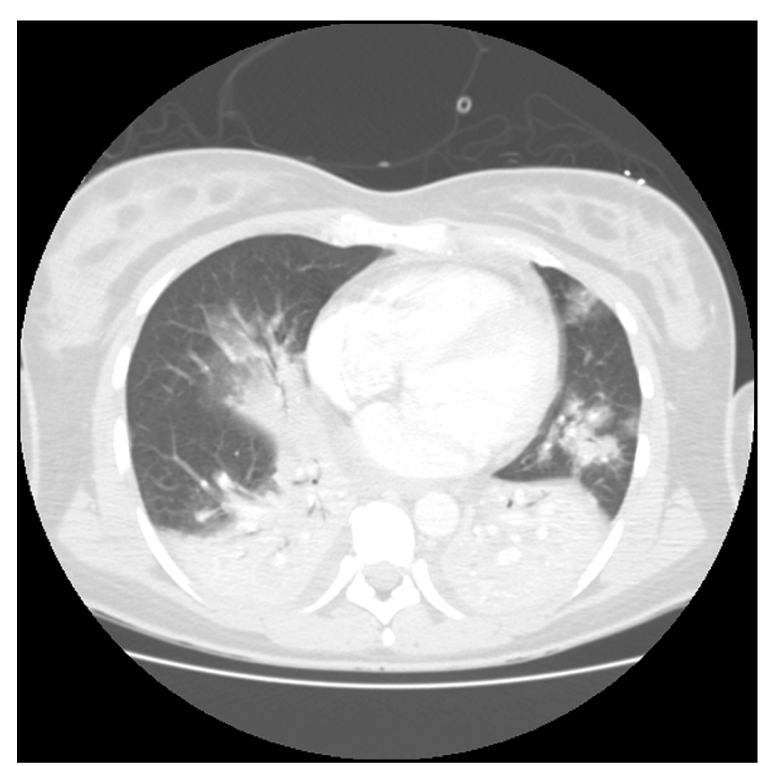

Figura 2. Tomografía computada de tórax muestra focos de condensación pulmonar bilaterales de predominio en lóbulos inferiores y derrame pleural bilateral escaso.

dada de alta una semana después, con indicaciones de control en el Consultorio de Enfermedades Respiratorias y Endocrinología.

\section{DisCUSIÓN}

El estreptococo beta hemolítico del grupo A (SGA) es una bacteria grampositiva que suele ser aislada en casos de faringoamigdalitis e infecciones de piel y tejidos blandos, y de forma excepcional es el agente causal de neumonías y bacteriemia $^{1-7}$. La mayoría de los casos son esporádicos y adquiridos en la comunidad $^{7-11}$. El microorganismo entra en el pulmón mediante inhalación o microaspiración, en raras ocasiones secundario a diseminación hematógena. La transmisión del SGA es por contacto directo con personas infectadas, que en general tienen faringitis. Pueden darse brotes en ambientes de hacinamiento $\mathrm{o}$ en guarderías y excepcionalmente pueden ser transmitidos por los alimentos ${ }^{5,6,13}$. El estado de portador de SGA es posible en un pequeño porcentaje de la población sana, es transitorio, y no es epidemiológicamente relevante. En nuestra paciente, no se identificaron factores de riesgo epidemiológico para adquirir esta infección, excepto el antecedente de la neoplasia tiroidea, y no se logró aislar el microorganismo en los cultivos de secreción faríngea que fueron tomados al tercer día de tratamiento antibiótico.

Los mecanismos patogénicos involucrados en la infección grave por $\mathrm{S}$ pyogenes han sido parcialmente esclarecidos, donde interactúan los mecanismos de defensa del huésped y los factores específicos de virulencia del microorganismo que son fundamentalmente la proteína $\mathrm{M}$ y las exotoxinas $^{14,15}$. La proteína $M$ es una proteína filamentosa de la membrana celular que presenta propiedades antifagocitarias, siendo los subtipos M 1, 3, 12 y 28 los más frecuentemente asociados a infecciones graves en pacientes con shock y falla multiorgánica. Se han identificado tres tipos de exotoxinas estreptocócicas: A, B y C. La exotoxina A (más frecuente en Estados Unidos de Norteamérica) y la B (más frecuente en Suecia y Reino Unido) se encuentran en la mayor parte de los pacientes con infecciones graves por $\mathrm{S}$ pyogenes. In vitro, las exotoxinas producen citotoxicidad, fiebre y aumentan los efectos de las endotoxinas, comportándose como superantígenos.

La presentación clínica y analítica es similar a la neumonía neumocócica ${ }^{2-4,7,16}$. Al-Kaabi y cols examinaron el cuadro clínico-radiográfico y la evolu- 
ción de la neumonía neumocócica y la neumonía por $\mathrm{S}$ pyogenes en población pediátrica ${ }^{3}$. Los pacientes con neumonía asociada a $\mathrm{S}$ pyogenes tuvieron derrames pleurales más extensos, mayor incidencia de cultivos positivos de líquido pleural, junto con un síndrome febril y período de internación más prolongados. La radiología es muy característica, con aparición de infiltrados bronconeumónicos en lóbulos inferiores que se complican a las pocas horas con derrame pleural en 55\%-95\% de los casos $3,4,7,16$. Algunos autores lo han denominado "pleuritis explosiva" por su rápida evolución ${ }^{17}$. El líquido pleural cumple criterios bioquímicos de exudado paraneumónico o de empiema, con tendencia a la formación de loculaciones muy precoces ${ }^{4,16,17}$. Los cultivos en el líquido pleural son positivos entre 20\% y 40\%. El tratamiento mediante tubo de drenaje torácico intercostal debe realizarse con la mayor brevedad, así como tratamiento antibiótico con penicilina intravenosa asociada a clindamicina, ante la sospecha diagnóstica y antes de recibir el estudio de sensibilidad $^{18,19}$. La infección pulmonar por SGA tiene letalidad elevada, entre $38 \%$ y $47 \% 0^{2-4,7,16}$. Por tanto se debe sospechar neumonía por SGA en presencia de afectación de lóbulos inferiores con desarrollo rápido y progresivo de derrame pleural, tal como ocurrió en nuestra paciente.

Los cambios en la epidemiología de las infecciones ocasionadas por $\mathrm{S}$ pyogenes se han relacionado estrechamente con la disponibilidad de antibióticos y la mejoría en el acceso a la atención médica para precisar con oportunidad el diagnóstico de las infecciones estreptocócicas. Aun cuando la penicilina es el antibiótico de elección para el tratamiento de las infecciones por SGA, la eritromicina o alguno de los nuevos macrólidos es la elección de segunda línea y de preferencia en pacientes con hipersensibilidad a la penicilina ${ }^{18,19}$. A diferencia de otras cocáceas Gram positivas, como Staphylococcus y Streptococcus pneumoniae, S pyogenes se ha mantenido susceptible a lo largo del tiempo a concentraciones muy bajas de penicilina, siendo éste el fármaco de elección; a pesar de su amplio uso por más de 50 años para el tratamiento de infecciones por este agente, no se han descrito hasta ahora cepas resistentes $^{18}$. En los últimos años, en muchos países se ha incrementado el uso de eritromicina y los nuevos macrólidos, promocionados para el tratamiento empírico de las infecciones respiratorias como otitis media, faringoamigdalitis, sinusitis y neumonía. En consecuencia ha habido un aumento alarmante en la resistencia del Streptococcus pyogenes a los macrólidos ${ }^{20}$. En esta bacteria se reconocen dos mecanismos de resistencia a macrólidos $^{21}$ : a) Modificación del sitio blanco: este mecanismo se produce por la adquisición de un gen erm (erythromycin ribosome methylase) que codifica una enzima que metila un residuo específico de adenina en el ARN ribosomal, produciendo un cambio conformacional en el ribosoma que disminuye la afinidad a macrólidos, lincosamidas y streptogramina B. Esta resistencia cruzada se conoce como resistencia fenotípica MLS $_{\mathrm{B}}$ y se expresa en forma constitutiva o inducible. b) Eflujo activo: este mecanismo se produce por la presencia de un gen mef (macrolide efflux) que codifica la síntesis de una bomba que media el eflujo en forma activa, sólo confiere resistencia a macrólidos, por lo que se conoce como resistencia fenotípica $M$, siendo el principal mecanismo de resistencia descrito en $\mathrm{S}$ pyogenes. En nuestro medio, la resistencia de $\mathrm{S}$ pyogenes a los macrólidos se ha mantenido bajo $10 \%^{22,23}$. En el Laboratorio Clínico de la Universidad Católica fueron aisladas entre 1990 y 1998, 594 cepas de S pyogenes, encontrándose 32 cepas $(5,4 \%)$ resistentes, correspondiendo $87,5 \%$ al fenotipo $\mathrm{M}$ y $12,5 \%$ al fenotipo $\mathrm{MLS}_{\mathrm{B}}{ }^{22}$. En el Laboratorio Clínico de Integramédica se aislaron 477 cepas entre 1996 y 2000 encontrándose 29 cepas $(6,1 \%)$ resistentes ${ }^{23}$.

En conclusión, la neumonía por S pyogenes es una entidad clínica infrecuente en el adulto, el cuadro clínico-radiográfico es similar a la neumonía neumocócica, la penicilina sigue siendo el tratamiento de elección y el riesgo de complicaciones supurativas (empiema, abscesos), falla respiratoria progresiva y shock séptico son significativos. 


\section{REFERENCIAS}

1. Carapetis JR, Steer AC, Mulholland EK, Weber M. The global burden of group A streptococcal diseases. Lancet Infect Dis 2005; 5: 685-94.

2. Kalima P, Riordan T, Berrisford RG, Sarsfield PT. Necrotizing pneumonia associated with group A streptococcal bacteraemia. Eur J Clin Microbiol Infect Dis 1998; 17: 296-8.

3. Al-Kaabi N, Solh Z, Pacheco S, Murray L, Gaboury I, LE SAUX N. A comparison of group A Streptococcus versus Streptococcus pneumoniae pneumonia. Pediatr Infect Dis J 2006; 25: 1008-12.

4. Keefer CS, Rantz LA, Rammelkamp CH. Hemolytic streptococcal pneumonia and empyema: a study of 55 cases with special reference to treatment. Ann Intern Med 1941; 14: 1533-50.

5. MacCallum WG. The pathology of the pneumonia in the United States Army camps during the winter of 1917-18. New York, NY: Rockefeller Institute for Medical Research; 1919. Monograph 10.

6. Crum NF, Russell KL, Kaplan EL, Wallace MR, Wu J, Ashtari P ET AL. Pneumonia outbreak associated with group A Streptococcus species at a military training facility. Clin Infect Dis 2005; 40: 511-8.

7. Birch C, Gowardman J. Streptococcus pyogenes: a forgotten cause of severe community-acquired pneumonia. Anaesth Intensive Care 2000; 28: 87-90.

8. Christie CD, Havens PL, Shapiro ED. Bacteremia with group A streptococci in childhood. Am J Dis Child 1988; 142: 559-61.

9. Demers B, Simor Ae, Vellend H, Schlievert PM, Byrne $\mathrm{S}$, JAMIESON F ET AL. Severe invasive group A streptococcal infections in Ontario, Canada: 1987-1991. Clin Infect Dis 1993; 16: 792-800.

10. Zurawski CA, Bardsley M, Beall B, Elliott Ja, Facklam $\mathrm{R}$, Schwartz B ET AL. Invasive group A streptococcal disease in metropolitan Atlanta: a population-based assessment. Clin Infect Dis 1998; 27: 150-7.

11. Davies HD, McGeer A, Schwartz B, Green K, Cann D, SimOR AE ET AL. Invasive group A streptococcal infections in Ontario, Canada. N Engl J Med 1996; 335: 547-54.

12. Stevens DL, Tanner MH, Winship J, Swarts R, Ries KM, SChlievert PM ET AL. Severe group A streptococcal infections associated with a toxic shock-like syndrome and scarlet fever toxin A. NEngl J Med 1989; 321: $1-7$.

13. Gamba ma, Martinelli M, Schaad HJ, Streuli RA, DiPersio J, Matter L et al. Familial transmission of a serious disease-producing group A streptococcus clone: case reports and review. Clin Infect Dis 1997; 24: 1118-21.

14. Cunningham MW. Pathogenesis of group A streptococcal infections and their sequelae. Adv Exp Med Biol 2008; 609: 29-42.

15. Tart AH, Walker MJ, Musser JM. New understanding of the group A Streptococcus pathogenesis cycle. Trends Microbiol 2007; 15: 318-25.

16. Barnham M, Weightman N, Anderson A, Pagan F, Chapman S. Review of 17 cases of pneumonia caused by Streptococcus pyogenes. Eur J Clin Microbiol Infect Dis 1999; 18: 506-9.

17. Braman SS, Donat WE. Explosive pleuritis. Manifestation of group A beta-hemolytic streptococcal infection. Am J Med 1986; 81: 723-6.

18. Horn DL, Zabriskie KB, Austrian R, Cleary PP, Ferretti JJ, Fischetti VA Et AL. Why have group A streptococci remained susceptible to penicillin? Report on a symposium. Clin Infect Dis 1998; 26: 1341-5.

19. Betriu C, Sánchez A, Gómez M, Cruceyra A, Picazo JJ. Antibiotic susceptibility of group A streptococci: a 6year follow-up study. Antimicrob Agents Chemother 1993; 37: 1717-9.

20. Seppälä H, Nissinen A, Järvinen H, Huovinen S, HenriksSON T, Herva E ET al. Resistance to erythromycin in group A streptococci. N Engl J Med 1992; 326: 292-7.

21. Ruof KL, Whiley RA, Beighton D. Streptococcus. En: Murray PR, Baron EJ, Pfaller MA, Tonover FC, Yolken RH, ed. Manual of Clinical Microbiology. $7^{\mathrm{a}}$ edición. Washington, D.C.: American Society for Microbiology 1999; 283-96.

22. Palavecino El, Riedel I, Berríos X, Bajaksouzian S, Johnson D, Kaplan E, Jacobs MR. Prevalence and mechanisms of macrolide resistance in Streptococcus pyogenes in Santiago, Chile. Antimicrob Agents Chemother 2001; 45: 339-41.

23. Camponovo R. Problemas de resistencia en Streptococcus pyogenes. Rev Chil Infect 2002; 19(Supl 2): S107-10. 